\title{
Localized Effects of Microwave Radiation on the Intact Eye Lens in Culture Conditions
}

\author{
A. Dovrat, ${ }^{1}$ R. Berenson, ${ }^{2}$ E. Bormusov, ${ }^{1}$ A. Lahav, ${ }^{2}$ T. Lustman, ${ }^{2}$ N. Sharon, ${ }^{1}$ and L. Schächter ${ }^{2 *}$ \\ ${ }^{1}$ Rappaport Faculty of Medicine Technion-Israel Institute of Technology, Haifa, Israel \\ ${ }^{2}$ Department of Electrical Engineering Technion-Israel Institute of Technology, \\ Haifa, Israel
}

\begin{abstract}
A novel experimental system was used to investigate the localized effects of microwave radiation on bovine eye lenses in culture for over 2 weeks. Using this setup, we found clear evidence that this radiation has a significant impact on the eye lens. At the macroscopic level, it is demonstrated that exposure to a few $\mathrm{mW}$ at $1 \mathrm{GHz}$ for over $36 \mathrm{~h}$ affects the optical function of the lens. Most importantly, self-recovery occurs if the exposure is interrupted. At the microscopic level, close examination of the lens indicates that the interaction mechanism is completely different from the mechanism-causing cataract via temperature increase. Contrary to the latter's effect, that is particularly pronounced in the vicinity of the sutures and it is assumed to be a result of local friction between the edges of the fibers consisting the lens. Even if macroscopically the lens has recovered from the irradiation, microscopically the indicators of radiation impact remain. Bioelectromagnetics 26:398-405, 2005.

(C) 2005 Wiley-Liss, Inc.
\end{abstract}

Key words: eye-lens exposure; cataract; sutures; athermal effect

\section{INTRODUCTION}

The need to establish a standard for exposure to microwave radiation occurred shortly after WWII, when it was realized that radar operators developed cataracts at a rate exceeding by far that of the overall population. Although, studies in the 60s and 70s have disputed this correlation [Cleary et al., 1965; Cleary and Pasternack, 1966; Appleton and McCrossen, 1972; Appleton et al., 1975], historically it was this concern that initially triggered the need to investigate the exposure to microwave radiation. In fact, experiments performed on animals confirmed the correlation between cataract and exposure to microwave radiation, facilitating the determination of the guidelines for exposure [ICNIRP, 1998]. Due to its natural sensitivity to radiation, the eye was the focus of many research programs part of which are reviewed by Lin [2003] and Elder [2003].

Using the eye as a measure of exposure to microwave radiation detector, researchers were able to establish the maximum levels of energy flux humans may be exposed to without increasing the probability of developing cataracts; for many years this was used as the only exposure criterion. Another accepted measure is the so-called specific absorption rate (SAR) representing the average power density absorbed in a given volume per average weight density [W/Kg or $\mathrm{mW} / \mathrm{g}]$; this absorbed power is assumed to be due to ionic conduction, which in turn may lead to temperature increase. A careful investigation of temperature variations due to absorption of microwave power within the eye and specifically in the closed vicinity of the lens has been performed by Guy et al. [1975] and Elson [1995], leading to the conclusion that for rabbits, $41^{\circ} \mathrm{C}$ seems to be the threshold for cataract to develop corresponding to $3{ }^{\circ} \mathrm{C}$ increase above the normal.

Bernardi et al. [1998] has reported simulation results of temperature increase in the eye at various frequencies $(6-30 \mathrm{GHz})$ using FTDT code with a spatial resolution of $0.5 \mathrm{~mm}$. Illuminating with a plane wave $\left(1 \mathrm{~mW} / \mathrm{cm}^{2}\right.$ at $\left.6 \mathrm{GHz}\right)$, they reported an increase of $0.04{ }^{\circ} \mathrm{C}$ in the lens-this increase is reduced for similar energy flux but at higher frequency. Similar

Grant sponsor: Rappaport Family Institute for Research in the Medical Sciences Technion V.P.R. fund; Grant sponsor: Edward S. Mueller Eye Research Fund; Grant sponsor: Guzik Ophthalmology Research Fund.

*Correspondence to: L. Schächter, Department of Electrical Engineering, Technion-IIT, Haifa 32000, Israel.

E-mail: levi@ee.technion.ac.il

Received for review 15 July 2004; Final revision received 18 January 2005

DOI 10.1002/bem.20114

Published online 10 May 2005 in Wiley InterScience (www.interscience.wiley.com). 
results were reported by Hirata et al. [2000] when scanning the range $0.6-6 \mathrm{GHz}$. As in many other electromagnetic problems, power absorption is dependent on the angle of incidence [Lin and Gandhi, 1996] as well as on the polarization of the wave [Hirata et al., 2000]. If this is not enough, Kramar et al. [1978] reported that monkeys did not develop cataract for the same energy flux as the one used for rabbits, a difference attributed [Elder, 2003] to the different facial structure of the two species.

There is no doubt today that microwave radiation via thermal effects may lead to cataract and the guidelines for exposure to microwave radiation reflect this fact [ICNIRP, 1998]. The question we intend to address here is whether athermal mechanism may affect the lens and lead to cataract on the long run. Furthermore, thermal effects are generally considered at the macroscopic scale (millimeters) and not microscopic scale (micrometers or smaller) therefore the natural question is, can processes occurring on this scale eventually lead to cataract although the global temperature increase is significantly below the $3{ }^{\circ} \mathrm{C}$ temperature increase believed to cause cataract [Guy et al., 1975]. In this context, athermal effects are conceived as electronic, vibration, or rotational resonance of a molecule, of a cell or even an entire biologic subsystem (e.g., nerve). In the framework of this brief report, we present clear evidence of two biophysical mechanisms: one is responsible to dramatic effects at the microscopic level and at least during the period tested (up to 15 days) there was no indication of recovery. The other, occurs at the macroscopic level and it manifests itself in reduced ability of the lens to focus a laser beam. Once the irradiation is interrupted, clear evidence of recovery is revealed.

\section{MATERIALS AND METHODS}

\section{Lens Organ Culture System}

Harnessing expertise developed [Dovrat et al., 1986; Sivak et al., 1986] to characterize the optical properties of intact bovine lens in long-term culture conditions, we developed a system to determine the effect of microwave radiation on the eye lens. Lenses were excised from eyes of 1-year-old male calves, obtained from a slaughterhouse; the lenses were incubated in culture within $2-4 \mathrm{~h}$. From each pair, one lens is exposed to microwave radiation and the lens from the other eye is used for control. All control lenses were kept in another incubator at the same temperature for the same period of time without any exposure to microwave radiation. At any given time, four lenses were exposed and their four counterparts are used as controls.
Each lens was placed in a specially designed culture chamber, completely immersed in culture medium. The latter consists of M199 with Earl's balanced salt solution, 3\% fetal calf serum and antibiotics (Penicillin $100 \mathrm{U} / \mathrm{ml}$ and Streptomycin $0.1 \mathrm{mg} / \mathrm{ml}$ ). All lenses were incubated at $35{ }^{\circ} \mathrm{C}$. Experimental treatments were initiated after preincubation of $24 \mathrm{~h}$. Only non-damaged pairs of lenses were included in the study.

A series of lenses was exposed to microwave radiation (typically $1.1 \mathrm{GHz}, 2 \mathrm{~mW}$ ). Each lens was exposed virtually around the clock: each hour it was exposed for a $50 \mathrm{~min}$ session followed by a $10 \mathrm{~min}$ break. During one of these breaks, every $24 \mathrm{~h}$, it was tested optically and compared to the control lens. During the short (5 min) optical test, the lens was not exposed to radiation, but when exposed, its average temperature was maintained constant in an incubator.

\section{Microwave System}

A computer controlled microwave source was built to feed the four transmission lines (Fig. 1) surrounding each vessel, each one in turn containing a single lens. The schematic diagram of the system is illustrated in Figure 2. It consists of a voltage-controlled oscillator (VCO) generating a constant microwave power $(13 \mathrm{dBm})$. Since it is necessary to control the radiation intensity the lenses are being exposed to the output from the VCO is passed through two attenuators: one is fixed and the other is variable, the latter being computer controlled, permitting the desired degree of freedom regarding the exposing intensity. In order to restore the power levels to the desired intensities, the microwave signal is directed into a $30 \mathrm{~dB}$ amplifier, which has a maximum output of $1 \mathrm{~W}$ before saturation occurs. A four-arm power splitter provides each transmission line with a microwave signal attenuated by $7 \mathrm{~dB}$ relative to the output of the amplifier. The signal traversing the transmission line is absorbed by a matched termination; the amplifier is well isolated from the transmission lines.

The transmission lines that guide the microwave field have consumed most of the design time in an attempt to generate the most uniform electric field possible in the region of the lens, taking into account the effect of the containing vessel and of course the surrounding fluid while allowing exposure of the lens to the maximal amount of power flowing in its vicinity. Since, the lens is exposed to a transverse electromagnetic (TEM) mode and its size is at least a factor of three smaller than the wavelength of interest in the medium $(\varepsilon \sim 42)$, the transverse distribution of the field was considered to be quasi-static. Further, it was assumed that the spatial field variations due to the different 


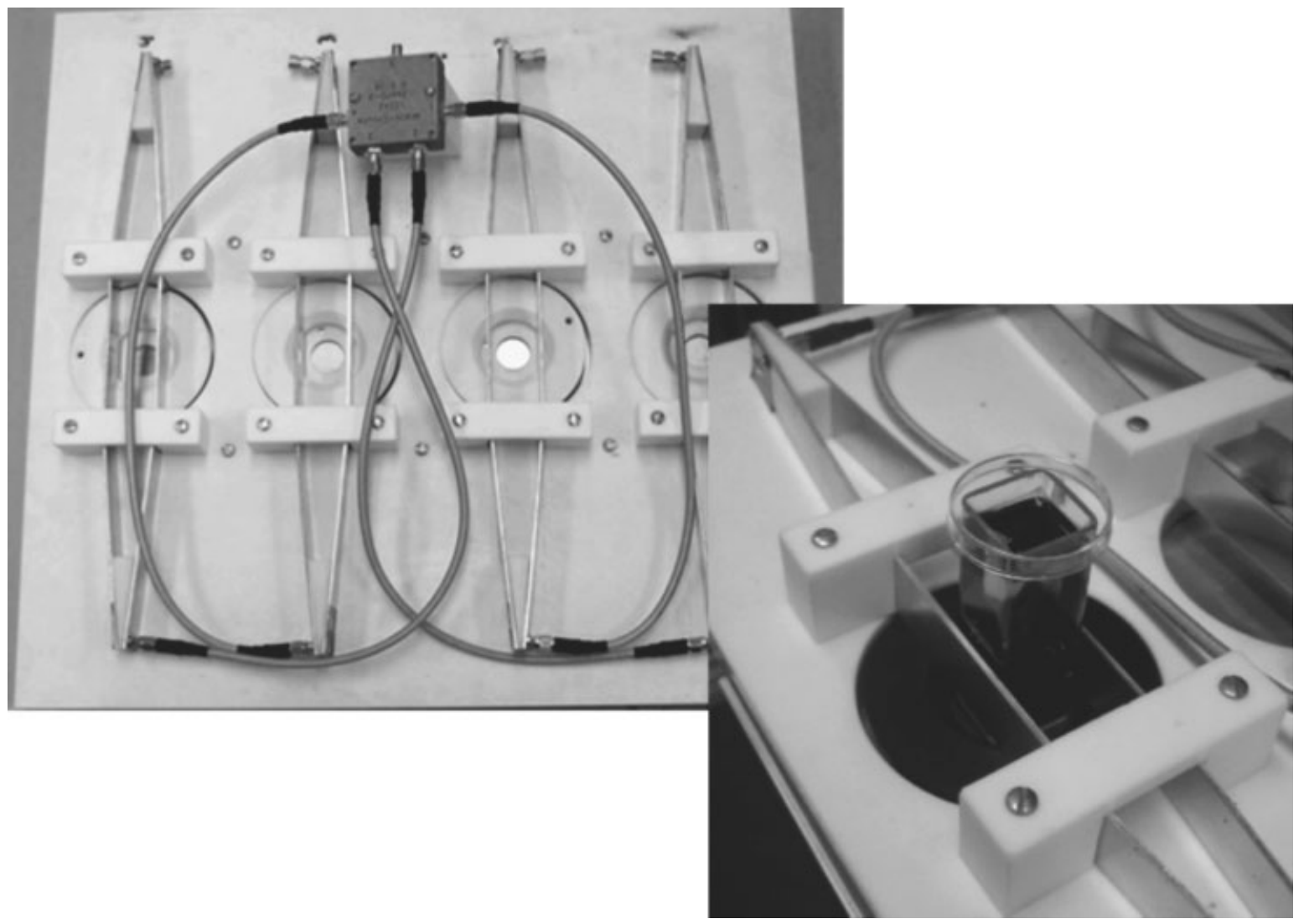

Fig. 1. The electromagnetic system exposing four eye lenses to electromagnetic radiation-left frame. Each vessel containing a lens, as the one illustrated in the right frame, is inserted between the two plates of the transmission line. The entire system is placed in an incubator maintaining constant temperature for the duration of exposure.

dielectric properties of the lens and the medium are negligible, when compared to the variations associated with the geometry of the vessel and the two metallic plates forming the transmission line. Figure 3 illustrates

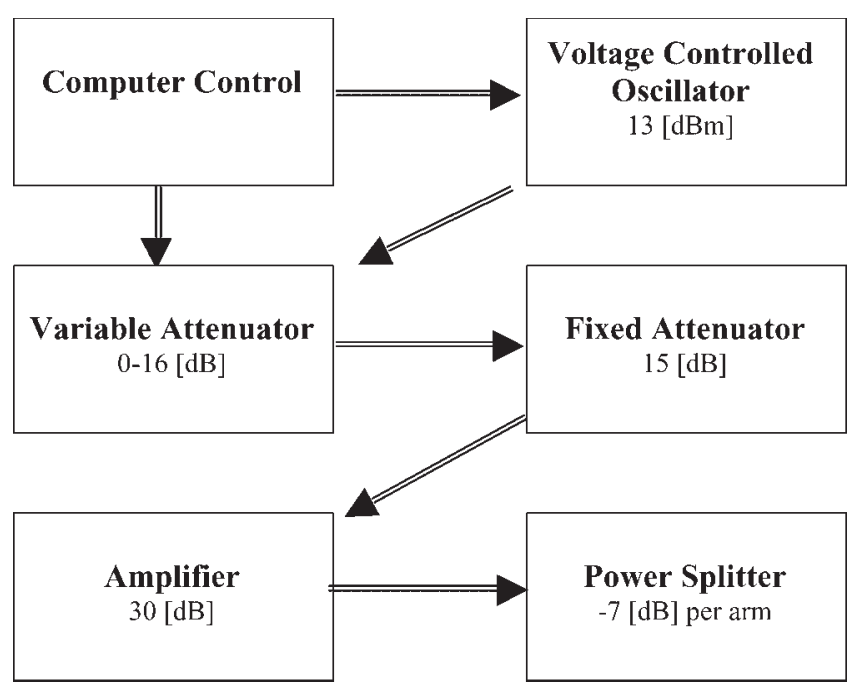

Fig. 2. Block diagram of the electromagnetic setup. the configuration, designed to maximize both the uniformity and the intensity of the field in the lens region (11-12 $\mathrm{mm}$ height and $15-16 \mathrm{~mm}$ diameter). In addition to these two constraints, it was necessary to design the system to be insensitive to small variations in the location of the containing vessel since this system is designed to operate over a relatively broad range of frequencies $(1-2 \mathrm{GHz})$. For this purpose, special attention was paid to the design of the tapers of the transmission line (see Fig. 1).

From the perspective of exposure of the lens to radiation, it is important to have a good estimate of the power traversing the lens. To perform this calibration, we measured the power at the end of the transmission line at the location of the termination in two cases: (1) when the vessel was in the transmission line, without the lens, but filled with the tissue culture liquid, and (2) when a metallic coin of the size of a lens was placed at the exact location of the lens. The difference between the two readings is the upper limit of what the lens may absorb. Calibration with the lens in place was performed too. Unless if otherwise specified, the incident power in all experiments reported here is 


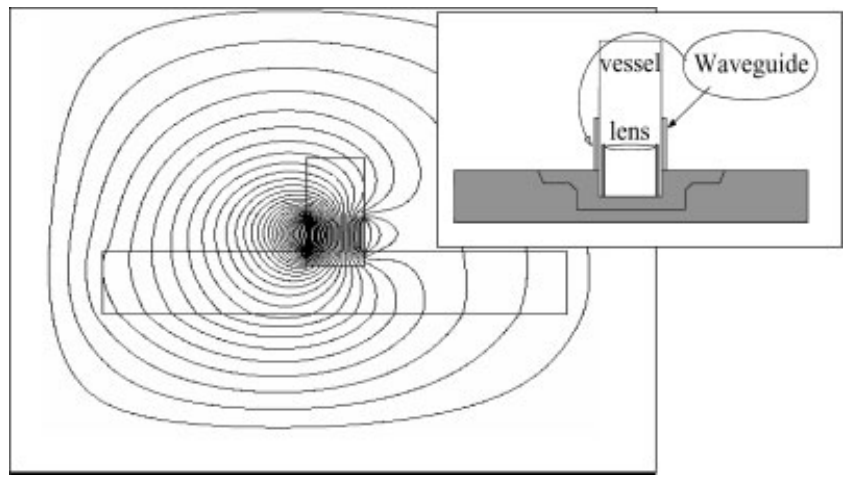

Fig. 3. Electrodes that are of the same width as their separation facilitate optimal uniformity and exposure. The plot illustrates the potential contours whereas the right frame shows a schematic of the system consisting of the vessel, waveguide (transmissionline), and Teflon holder; water $\left(\varepsilon_{r} \sim 42\right)$ fills the vessel up to the height of the metallic plate consisting the transmission line. Denoting the energy density in the region of interest by $w(\vec{r})=\frac{1}{2} \varepsilon_{0} \varepsilon_{r} \vec{E} \cdot \vec{E}$ thus the total energy $\langle w\rangle=\frac{1}{V} \int \mathrm{d} V w(\vec{r})$ in this volume is proportional to the average energy density and our measure for uniformity is the standard deviation $\Delta w^{2}=\frac{1}{V} \int \mathrm{d} V[w-\langle w\rangle]^{2}$. In the design process, we aimed to a typical ratio $\Delta w /\langle w\rangle$ of less than 0.1 .

$2.2 \mathrm{~mW}$. Since the weight of a lens is typically $1.6 \mathrm{~g}$, the maximum SAR in our experiment is of the order of $1.4 \mathrm{~W} / \mathrm{Kg}$, which is twice the limit of the allowed exposure in this range of frequencies. In order to put our study in an adequate perspective, it is important to indicate that earlier experiments with rabbits have shown [Guy et al., 1975] that the threshold intensity radiation for cataract to occur when irradiated with $2.45 \mathrm{GHz}$ radiation for $100 \mathrm{~min}$ is $1.5 \mathrm{~kW} / \mathrm{m}^{2}$, corresponding to a SAR of $130 \mathrm{~W} / \mathrm{kg}$ peak absorption. In our case the energy flux is one order of magnitude lower, namely, $0.1 \mathrm{~kW} / \mathrm{m}^{2}$ at $1.1 \mathrm{GHz}$; however, the lens is exposed for 8 days. For comparison, the guideline for exposure [ICNIRP, 1998] is $0.05 \mathrm{~kW} / \mathrm{m}^{2}$.

\section{Temperature Measurement}

Another topic that was carefully examined is the temperature. It is the purpose of this study to focus on effects that are not caused by global temperature increase. Therefore, it was important to establish that the temperature does not change as a result of the electromagnetic energy injected into the system. A series of temperature measurements in the tissue culture fluid in the vicinity of the lens inside the vessel, were performed and the temperature was found to be stable and consistent with the temperature monitored by the incubator probe $\left(35^{\circ} \mathrm{C}\right)$. Throughout these tests the microwave power levels were maintained at the tested value.

To be more specific, the system was operated for 15 days with a metallic probe of YSI PRECISION
4000 A thermometer with a dynamic range between 0 and $50{ }^{\circ} \mathrm{C}$ (YSI Incorporated Yellow Springs Instrument Co. Inc. Yellow Springs Ohio, 45387). The probe has a disk-shaped (5 $\mathrm{mm}$ diameter) stainless steel tip with epoxy on one side of the disk connected to a 10 foot long, vinyl jacketed lead. The disk was located about 4 $\mathrm{mm}$ from the lens and for the entire period the measured temperature was in excellent agreement with the measurement of the incubator's probe. The prescribed accuracy of the monitoring probe is $0.1^{\circ} \mathrm{C}$ in the range between 32 and $42{ }^{\circ} \mathrm{C}$. It should be emphasized that for the available mass and for the potential power that may be converted to heat and bearing in mind the ability to dissipate the heat, the anticipated increase in temperature is miniscule and it is definitely less than the accuracy of our probe. Moreover, the probe accuracy is above the value that may allow us to measure a temperature increase predicted, albeit in a completely different configuration, by Bernardi et al. [1998] to be $0.04{ }^{\circ} \mathrm{C}$.

\section{Optical Monitoring System}

Bovine lenses (7-month-old calves) were incubated in a long term organ culture system for 15 days. Each lens was placed in a specially designed culture chamber [Dovrat et al., 1986]. Each lens was located in the culture chamber, leaving a clear space filled with culture medium both below and above the lens; this medium was changed daily. An optical bench [Sivak et al., 1990] was used for the daily test of both exposed and control lenses. A $670 \mathrm{~nm}$ diode laser with the beam parallel to the axis of the lens was directed towards the cultured lens along one meridian in $0.5 \mathrm{~mm}$ increments. After passing through the lens, the laser beam is refracted and the system determines the back vertex focal length for every beam position. Each scan consists of measurements of the same beam from 22 different points across the lens.

A lens of good optical quality is able to focus the laser beam from the various locations (see thin solidline in the left frame of Fig. 4). When the lens is damaged due to exposure to microwave radiation, its ability to focus the laser beam at the various locations is altered, as clearly revealed by the right frame of Figure 4. The thick dashed line connects the points of the back vertex distance for each ray passing through the lens. The thick solid line shows the relative intensity of each beam, that is, the transmitted intensity normalized to the incident one.

\section{RESULTS AND DISCUSSION}

Figure 5 summarizes the optical results of 20 control lenses and 20 lenses exposed to 192 cycles of 

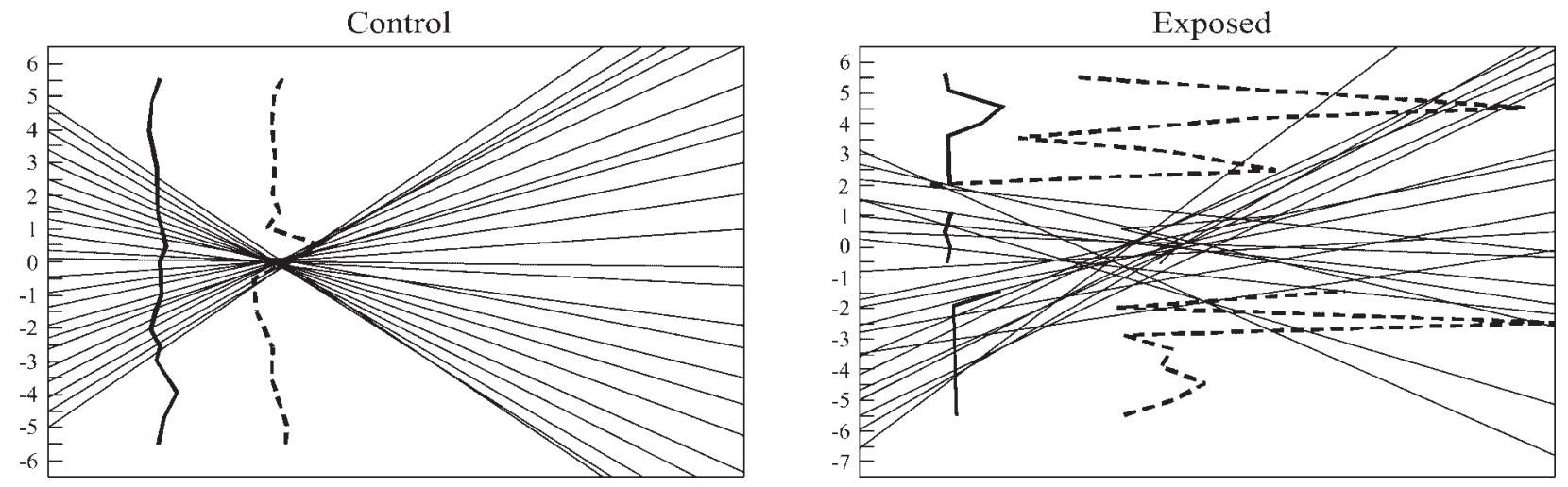

Fig. 4. Left frame: Good quality lens as demonstrated by the optical scanner. All rays passing through the lens have similar focal length. The thick dashed line connects the points of the back vertex distance for each ray passing through the lens. The thick solid line shows the relative intensity of each beam. Right frame: Exposed lens, showing considerable variability in the focal length of the beams passing through the lens.

electromagnetic radiation; as indicated earlier, each cycle lasted $50 \mathrm{~min}$ followed by a $10 \mathrm{~min}$ break. Focal length variability represents the variation in the 22 focal length measurements during each scan as shown in Figure 4 . The focal length variability is measured as the standard error (SE) of the focal length.

Control lenses show almost no variation in focal length during the 15 days of the culture. However, lenses exposed to microwave radiation show variation in focal length (damage) starting from about $48 \mathrm{~h}$ after initial exposure to the microwave radiation cycles. Four

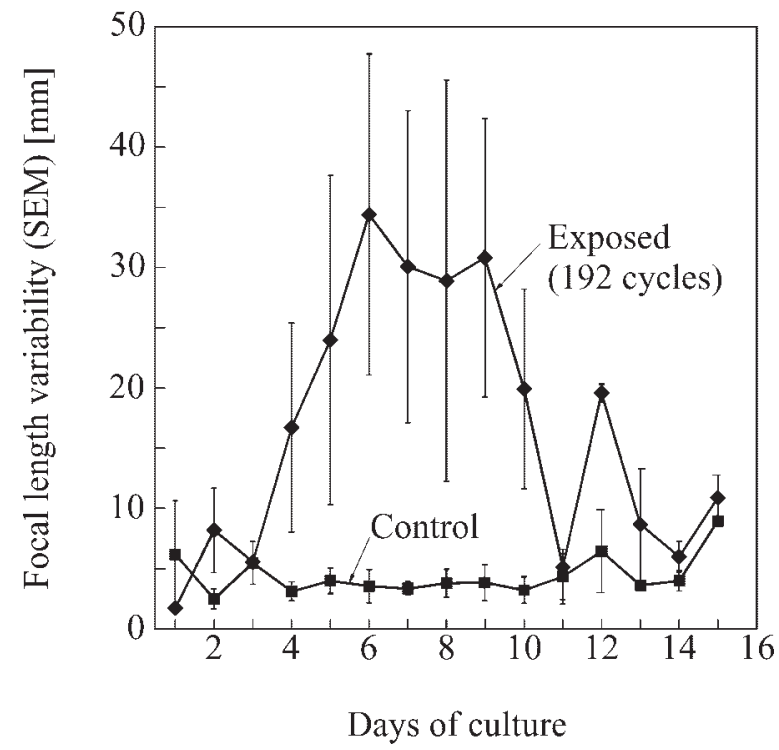

Fig. 5. Lens optical quality during 15 days of culture. Control lenses with almost no variation in focal length during 15 days of culture. Exposed lenses shows increase in focal length variability (optical damage) about $48 \mathrm{~h}$ after beginning of irradiation cycles. The damage was reduced when the irradiation stopped, after day 9 of the culture $(1.1 \mathrm{GHz}, 2.22 \mathrm{~mW})$. days later the optical damage reached its maximum with no further significant change up to the ninth day of culture. In this day, the radiation exposure (192 cycles) ends. Our optical test clearly reveals that the lens starts to recover, which is reflected by the reduced focal length variability. At the end of the experiments on days 12 or 16 of the culture, lenses were taken for analysis using an inverted microscope.

Before proceeding it is important to clarify our choice of SAR, which is about twice the recommended guidelines whereas the energy flux used is by more than a factor of 10 lower than values reported to cause cataract and twice larger than the recommended guidelines [ICNIRP, 1998]. This choice was dictated by our present ability to culture the lens for only up to 30 days and the need to have reliable results during this period. At less than half the intensity $(0.89 \mathrm{~mW})$, the reaction time of the system was almost double. In other words, it takes about 8 days to reach the level of $4 \mathrm{~mm}$ focal length variability, whereas the recovery time is virtually identical with that revealed in Figure 5. This result enlightens the role of the overall energy to which the lens is exposed during extended period of time.

Therefore, one can not rule out the possibility that by exposing the lens to radiation levels corresponding to SAR smaller than the guidelines [ICNIRP, 1998] will lead to similar effects on the focal length, provided the exposure occurs on time scales of many weeks. Moreover, these results suggest that the specific energy absorption (SA), defined as the energy density absorbed in the tissue divided by its weight density, should play a more important role in determining the guidelines for exposure. Further support to this conjuncture may be found in a work reported by Creighton et al. [1987] indicating that even when the energy is kept constant, but the dose and the duty cycle are changed, the 
radiation effect of a pulsed exposure is stronger than for a continuous case. Although, the recovery ability demonstrated above is encouraging, within the framework of this study, we are unable to determine its long term (many weeks or months) behavior due to the limited period of time we can culture the lens in a reliable manner.

The crystalline lens has unique morphological features: throughout the life span of the lens it is growing due to continuous addition of fiber cells. The ends of all fibers are interlocked to form the lens sutures. These are Y shaped [Kuszak et al., 1984] and their width varies across the lens; see top frame of Figure 6 illustrating a control lens. The bottom frame of Figure 6 reveals a profound effect of microwave radiation on bovine lens along the sutures as recorded after 12 days in culture under the same exposure parameters presented above $(1.1 \mathrm{GHz}, 2.22 \mathrm{~mW}, 192$ cycles, each cycle lasts for $50 \mathrm{~min}$ followed by a $10 \mathrm{~min}$ break). When comparing to the control lens, the most remarkable feature is the occurrence of "bubbles" at the sutures. It is further convenient to compare this result to the one in the absence of microwave radiation, when the bubbles are generated by temperature increase to $39.5{ }^{\circ} \mathrm{C}$ during $4 \mathrm{~h}$ (middle frame, Fig. 6). Clearly, their concentration is by far larger peripherally and diminishes inwards. In fact, at the point where two bundles of fibers meet (sutures), the temperature increase seems to be negligible, indicating poor thermal conductivity and heat capacity and alluding therefore to low electric conductivity.

As of now, we do not have a clear biophysical picture of the character of the mechanism responsible to this result, and it will require some further electrodynamic and thermal analysis. At this point, we can offer only a qualitative explanation to this result: Since, we deduced that the local electric conductivity of the sutures is relatively small, it is reasonable to anticipate that a different mechanism is responsible to the occurrence of the bubbles when the lens is exposed. Moreover, whatever the character of this mechanism is, it ought to be local. In order to envision what such a mechanism may be, let us focus our attention on the microscopic scale at the edges of the fibers forming the lens. These may vibrate under the effect of the microwave radiation and the "microscopic friction" at the interface between two bundles causes a local temperature increase. Consequently, the bubbles occur only at the interface between two bundles namely, the sutures. This picture, as seen in the bottom frame of Figure 6, has occurred in all lens exposed to microwave radiation and rarely (less than 30\%) in control lens, indicating that the calf, while alive, may have been exposed to an electromagnetic field.
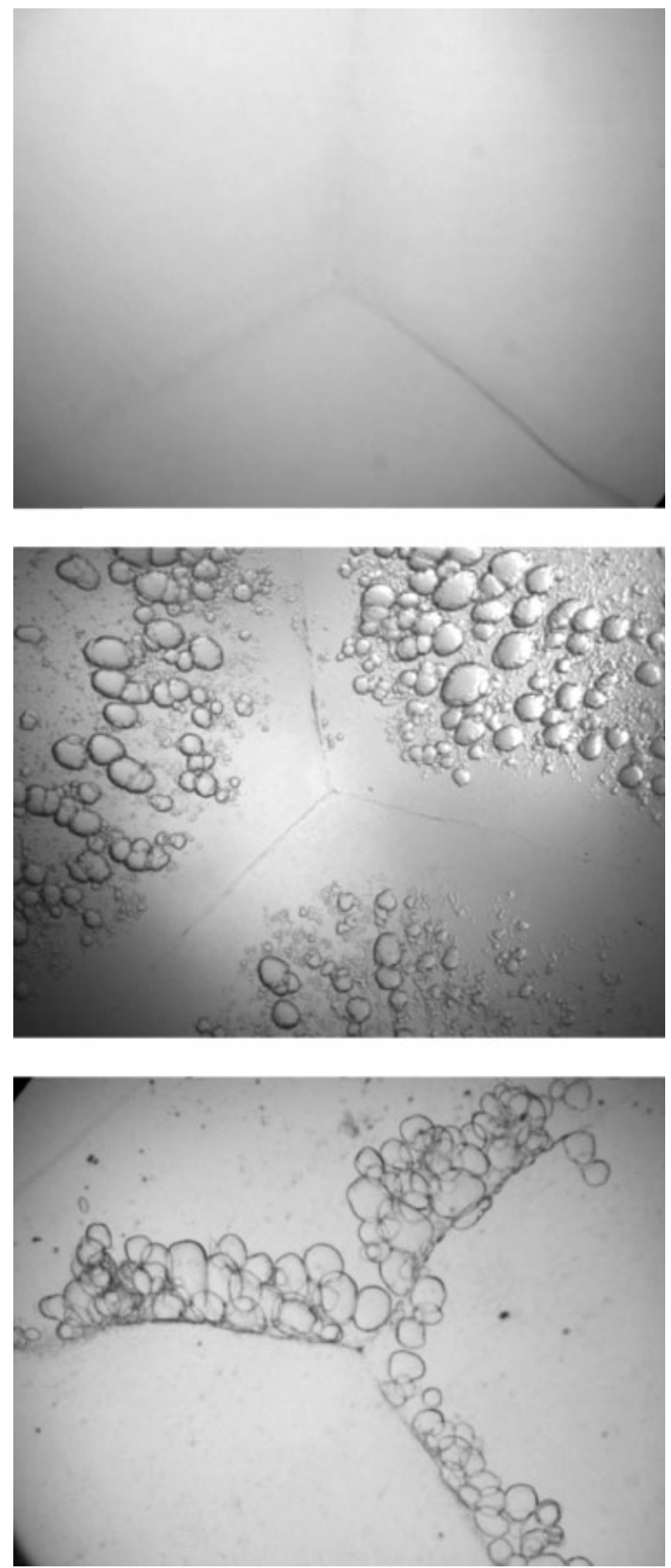

Fig. 6. Inverted microscope photographs of lenses incubated in organ culture conditions for 12 days. Control lens shows lens sutures with no damage - top frame. Bottom frame demonstrates the effect of microwave radiation on bovine lens sutures for a total exposure of 192 cycles $(1.1 \mathrm{GHz}, 2.22 \mathrm{~mW})$. Each cycle lasts $50 \mathrm{~min}$ followed by 10 min pause. In the absence of microwave radiation, the bubbles are generated by temperature increase to $39.5^{\circ} \mathrm{C}$ during $4 \mathrm{~h}$; see middle frame. 
It is important to re-emphasize that this experiment was performed at $1.1 \mathrm{GHz}$, and conceptually we do not anticipate significant impact(s) of this result except the ones associated with the different intensity linked to field distribution due to mode excitation and/ or field penetration. However, this is obviously beyond the scope of this study. The impact of the illumination intensity was the scope of several of the studies mentioned above. The one, which is the closest to the one presented here, is the study of Creighton et al. [1987] briefly mentioned above. They examined the depth of damage caused by pulsed as well as continuous microwaves as estimated by scanning electron microscopy in rat lenses fixed immediately, after irradiation in vitro in circulating thermostatically controlled buffered saline. Pulses of $10 \mu \mathrm{sec}$ duration and $24 \mathrm{~kW}$ peak power were delivered to the lens at different repetition rates in order to permit the same total energy to be delivered during 6,20 , or $60 \mathrm{~min}$ of irradiation at SAR values of $5.75,11.5,23,69,231$, and $750 \mathrm{~mW} / \mathrm{g}$; total energy deposited in the lens was $0.23,0.46$, $1.38,4.6$, and $15 \mathrm{~W} \mathrm{min/g}$. For comparison, our exposure lasted for up to 15 days, with a SAR of less than $1.5 \mathrm{~mW} / \mathrm{g}$.

The depth of degeneration when either pulsed or continuous signals are applied has been compared. For a given total energy delivered, the pulsed irradiation resulted in 4.7 times more damage than a continuous signal. Damage, revealed as granular degeneration of cells at the lens equator, was measured at the apex of penetration of the degeneration. In addition to surface granulation the damage was measured as (1) holes within the fiber cells, especially in the region of zonular attachment in the equatorial region; (2) globular degeneration, covering large sub-capsular regions, but mainly within the zonular attachment and equatorial region; (3) foam located immediately sub-capsularly within the same region; and (4) granulation of fiber cells which can extend deep within the lens. Delivering the same amount of energy in several modes (different "duty-cycles") and obtaining different levels of damage, they suggested that the mechanism responsible for the damage is pressure waves induced in the aqueous medium and lens tissue by thermo-acoustic expansion following each microwave pulse [Lin, 1978].

In conclusion, microwave radiation has a clear impact on the eye lens even when the temperature is maintained globally constant at $35^{\circ} \mathrm{C}$. According to our experimental results, there are at least two different mechanisms by which microwave radiation affects the eye lens.

1. Exposure beyond a certain energy level affects the optical transmission function of the lens, indicating some damage to the lens. This damage in turn has two important features: it saturates after a few days (4 days of exposure at $2.22 \mathrm{~mW}$ and 8 days of exposure at $0.89 \mathrm{~mW}$ ) and it virtually disappears once the irradiation is terminated.

2. Even after the lens has recovered optically (after the 12th day of culture), we detected microscopic effects (bubbles) along the lens sutures. This interaction mechanism is completely different from the mechanism-causing cataract via regular temperature increase and in particular, this difference is pronounced in the vicinity of the sutures.

Finally, in the context of the energy deposited, these results, which are consistent with those reported in Creighton et al. [1987] study, hint that more attention should be paid when determining the guidelines for exposure to the so-called (SA) and not only for energy flux and the SAR.

\section{ACKNOWLEDGMENTS}

This study was supported in part by a grant from the Rappaport Family Institute for Research in the Medical Sciences Technion V.P.R. fund-Edward S. Mueller Eye Research Fund and by Guzik Ophthalmology Research Fund. One of the authors (A.D.) thank Dr. Yair Weiss, Dr. Samuel Fruchter, Dr. Yuval Argov, and Dr. Eyal Tadmor for assistance with the study animals. One of the authors (L.S.) has benefited from enlightening comments of Dr. James Lin.

\section{REFERENCES}

Appleton B, McCrossen GC. 1972. Microwave lens effects in humans arch. Ophthalmologv 88:259-262.

Appleton B, Hirsh S, Kinion RO, Soles M, McCrossen GC, Neidlinger RM. 1975. Microwave lens effects in humans. Arch Ophthalmol 93:257-258.

Bernardi P, Cavagnaro M, Pisa S, Piuzzi E. 1998. SAR distribution and temperature increase in an anatomical model of the human eye exposed to the field radiated by the user antenna in a wireless LAN. IEEE Trans Microw Theory Tech 46:2074.

Cleary SF, Pasternack SB. 1966. Lenticular change in microwave workers: A Statistical Study. Arch Environ Health 12:23-29.

Cleary SF, Pastemack SB, Beebe GW. 1965. Cataract incidence in radar workers. Arch Environ Health 11:179-182.

Creighton MO, Larsen LE, Stewart-DeHaan PJ, Jacobi JH, Sanwal M, Baskerville JC, Bassen HE, Brown DO, Trevithick JR. 1987. In vitro studies of microwave-induced cataract. II. Comparison of damage observed for continuous wave and pulsed microwaves. Exp Eye Res 45:357-373.

Dovrat A, Sivak JG, Gershon D. 1986. Novel approach to monitoring lens function during organ culture. Lens Res 3:207-215.

Elder JA. 2003. Ocular effects of radiofrequency energy. Bioelectromagnetics Suppl 6:148-161. 
Elson E. 1995. Biological effects of radio-frequency and microwave fields: In vivo and in vitro experimental results. In: Editor in Chief Joseph D, editor. The biomedical engineering handbook. Bronzino, Bora Raton Florida, USA: CRC Press. 1417p.

Guy AW, Lin JC, Kramar PO, Emery A. 1975. Effect of $2450 \mathrm{MHz}$ radiation on the rabbit eye. IEEE Trans Microw Theory Tech 23:492-498.

Hirata A, Matsuyama S, Shiozawa T. 2000. Temperature rises in the human eye exposed to EM waves in the frequency range 0.6-6 GHz. IEEE Trans Electromagn Compatability 42: 386.

International Commission on Non-Ionizing Radiation Protection (ICNIRP). 1998. Guidelines for limiting exposure to time varying electric, magnetic and electromagnetic fields (up to $300 \mathrm{GHz}) ; 1998$. Health Phys 74:494-522.

Kramar P, Harris C, Emery AF, Guy AW. 1978. Acute microwave irradiation and cataract formation in rabbits and monkeys. J Microw Power 13:239-249.
Kuszak JR, Bertram BA, Macsai MS, Rae JL. 1984. Sutures of the crystalline lens: A review. Scan Electron Microsc Part 3: 1369-1378.

Lin JC. 1978. Microwave auditory effects and applications Thomas: Springfield Ill.

Lin JC. 2003. Cataracts and cell-phone radiation. IEEE Antennas and Propagation 45:171.

Lin JC. 1986. Computer methods for predicting field intensity. In: Polk C, Postow E, editors. Handbook of biological effects of electromagnetic fields, 2nd edition. Boca Raton, FL: CRC Press. pp. 273-313.

Sivak JG, Gershon D, Dovrat A, Weerheim J. 1986. Computer assisted scanning laser monitor of optical quality of the excised crystalline lens. Vision Res 26:1873-1879.

Sivak JG, Yoshimura M, Weerheim J, Dovrat A. 1990. Effect of hydrogen peroxide, DL-propranolol, and prednisone on the bovine lens optical function in culture. Invest Ophthalmol Vis Sci 31:954-963. 